\title{
Curcumin use in ulcerative colitis: is it ready for prime time? A systematic review and meta-analysis of clinical trials
}

\author{
Saurabh Chandan ${ }^{\mathrm{a}}$, Babu P. Mohan ${ }^{\mathrm{b} *}$, Ojasvini C. Chandan', Rizwan Ahmad", Abhishek Challa \\ Hemachand Tummalae, Shailender Singha ${ }^{a}$, Punita Dhawand, Suresh Ponnada ${ }^{f}$, Amar B. Singh ${ }^{d}$, \\ Douglas G. Adler ${ }^{9}$
}

University of Nebraska Medical Center, Omaha, Nebraska; University of Arizona, Banner University Medical Center, Tucson, Arizona; South Dakota State University, Brookings, South Dakota; Carilion Roanoke Memorial Hospital, Roanoke, Virginia; University of Utah School of Medicine, Salt Lake City, Utah, USA

\section{Abstract}

\begin{abstract}
Background Curcumin, an active ingredient of the Indian herb turmeric (Curcuma longa), has shown promising anti-inflammatory properties. Studies of its potential benefits in treating patients with ulcerative colitis (UC) are limited. We performed a systematic review and meta-analysis of human randomized placebo controlled trials to evaluate the efficacy of adjunctive therapy with curcumin in treating patients with UC.
\end{abstract}

Methods We conducted a search of several databases (from January 2000 to September 2018). A randomeffects model was used for analysis. We assessed heterogeneity between study-specific estimates using the Cochran Q statistical test, 95\% prediction interval (PI) and $I^{2}$ statistics. The outcomes assessed were the pooled odds of clinical response and remission as well as the endoscopic response.

Results A total of 7 studies with 380 patients (curcumin $n=188$; placebo $n=190$ ) were included in the final analysis. The pooled odds ratio for clinical remission with curcumin use was 2.9 (95\%CI 1.5-5.5, $\left.I^{2}=45, \mathrm{P}=0.002\right)$, clinical response was 2.6 (95\%CI $\left.1.5-4.5, I^{2}=74 \%, \mathrm{P}=0.001\right)$, and endoscopic response/remission was 2.3 (95\%CI 1.2-4.6, $\left.I^{2}=35.5 \%, \mathrm{P}=0.01\right)$.

Conclusions Based on our study, combined mesalamine and curcumin therapy was associated with roughly threefold better odds of a clinical response compared to placebo, with minimal side effects. This response was statistically significant, albeit with heterogeneity, probably due to the different severity scoring indices, curcumin dosages and routes of drug delivery used.

Keywords Ulcerative colitis, curcumin, meta-analysis

Ann Gastroenterol 2020; 33 (1): 1-9

\begin{abstract}
${ }^{\mathrm{a}}$ Gastroenterology and Hepatology, University of Nebraska Medical Center, Omaha, Nebraska (Saurabh Chandan, Abhishek Challa, Shailender Singh); ' Internal Medicine, University of Arizona, Banner University Medical Center, Tucson, Arizona (Babu P. Mohan);

'Pediatric Gastroenterology, Hepatology and Nutrition, University of Nebraska Medical Center, Omaha, Nebraska (Ojasvini C. Chandan); ${ }^{\mathrm{d} B i o c h e m i s t r y}$ and Molecular Biology, University of Nebraska Medical Center, Omaha, Nebraska (Rizwan Ahmad, Punita Dhawan); ${ }^{\mathrm{e}}$ College of Pharmacy and Allied Health Professions, South Dakota State University, Brookings, South Dakota (Hemachand Tummala); Internal Medicine, Carilion Roanoke Memorial Hospital, Roanoke, Virginia (Suresh Ponnada); ${ }^{\mathrm{B} D i v i s i o n}$ of Gastroenterology and Hepatology, University of Utah School of Medicine, Salt Lake City, Utah (Douglas G. Adler), USA

${ }^{\star}$ Saurabh Chandan and Babu P. Mohan contributed equally to the manuscript

Conflict of Interest: None

Correspondence to: Douglas G. Adler, MD, Gastroenterology and Hepatology, University of Utah School of Medicine, Huntsman Cancer Center 30N 1900E 4R118, Salt Lake City, Utah 84132, USA, e-mail: Douglas.adler@hsc.utah.edu
\end{abstract}

Received 8 August 2019; accepted 21 October 2019; published online 29 November 2019

DOI: https://doi.org/10.20524/aog.2019.0439

๑ 2019 Hellenic Society of Gastroenterology

\section{Introduction}

Ulcerative colitis (UC) is a chronic inflammatory condition that presents with watery and/or hemorrhagic diarrhea associated with rectal urgency. Symptoms can be debilitating and severely affect a person's quality of life. At diagnosis, 30$50 \%$ of patients have disease confined to the rectum or the sigmoid colon (distal colitis), 20-30\% have left-sided colitis and about $20 \%$ have pancolitis [1]. Of the patients with distal colitis, $25-50 \%$ progress to more extensive forms of the disease over time [2].

In early 2019, the American Gastroenterology Association (AGA) released clinical guidelines for the management of mild to moderate UC. The recommendation is to start standard dose mesalamine (2-3 g/day) or diazo-bonded 5-amino-salicylic acid (5-ASA), rather than low dose mesalamine, sulfasalazine or no treatment, in patients with extensive mild-moderate UC. The addition of rectal mesalamine to oral 5-ASA is recommended for patients with extensive or left-sided mildto-moderate UC [3].

There have been reports regarding the efficacy of curcumin, a natural phenol found in the large-leafed herb 
Curcuma longa L. (common names turmeric, Indian saffron) in the treatment of various diseases, such as hyperlipidemia, diabetes mellitus and non-alcoholic steatohepatitis, as well as UC [4-6]. However, the AGA made no recommendations on its use in mild-to-moderate UC patients already on a 5-ASA agent. The reason for this was stated to be a "knowledge gap", probably from the lack of large randomized placebo controlled studies (RCTs) evaluating the efficacy of curcumin and its side-effect profile. We therefore aimed at filling this "knowledge gap" by performing a systematic review and meta-analysis of the current evidence in order to evaluate the role of combination curcumin therapy in patients with UC.

\section{Materials and methods}

\section{Search strategy}

We conducted a comprehensive search of several databases and conference proceedings, including PubMed, EMBASE, Google Scholar, SCOPUS and Web of Science databases, for publications from January 2000 to September 2018. We followed the Preferred Reporting Items for Systematic Reviews and Meta-Analyses (PRISMA) guidelines [7], using a predefined protocol to identify studies reporting on the use of curcumin in UC. An experienced medical librarian using inputs from the study authors helped with the literature search.

Key words used in the literature search included a combination of "curcumin", "turmeric", "inflammatory bowel disease", and "ulcerative colitis". The search was restricted to studies in human subjects published in the English language in peer-reviewed journals. Two authors (BPM, SC) independently reviewed the title and abstract of studies identified in the primary search and excluded studies that did not address the research question, based on pre-specified exclusion and inclusion criteria. The full text of the remaining articles was reviewed to determine whether it contained relevant information. Any discrepancy in article selection was resolved by consensus, and in discussion with a coauthor. The bibliographic sections of the selected articles, as well as the systematic and narrative articles on the topic were manually searched for additional relevant articles.

\section{Study selection}

In this meta-analysis, we included clinical trials that evaluated the clinical outcomes of curcumin in UC. Studies were included as long as they provided data needed for the analysis, irrespectively of the sample size, inpatient/outpatient setting and geography. Only RCTs reporting the efficacy of curcumin in UC were included in this meta-analysis. Exclusion criteria comprised: 1) case reports and case series; and 2) studies not published in English. In the event of multiple publications from the same cohort and/or overlapping cohorts, data from the most recent and/or most appropriate comprehensive report were retained.

\section{Data abstraction and quality assessment}

Data on study-related outcomes in the individual studies were abstracted onto a standardized form by at least 2 authors (SC, OCC), and 2 authors (BPM, SC) did the quality scoring independently. The Jadad scale for RCTs was used to assess the quality of studies, the details of which are provided in Supplementary Table 1 [8].

\section{Definitions}

The response of UC to treatment was assessed using the following indices: Clinical Activity Index (CAI); Simple Clinical Colitis Activity Index (SCCAI); and Disease Activity Index (DAI).

The CAI indexing system comprises 7 items: stool frequency (0-3); blood in stool (0-4); general well-being (0-3); abdominal discomfort (0-3); fever (0-3); extraintestinal manifestations (0-9); and laboratory findings (erythrocyte sedimentation rate and hemoglobin) (0-4) [9].

The SCCAI system comprises 6 items: bowel frequency during the day (0-3); bowel frequency at night (1-2); urgency of defecation (1-3); blood in stool (1-3); general well being (0-4); and extra-colonic features (1 per manifestation) [10].

The DAI, or Mayo score, first developed in 1987, calculates a score between 0 and 12 and includes assessment of stool frequency, rectal bleeding, findings of flexible proctosigmoidoscopy and physician's global assessment of disease activity [11]. The Mayo endoscopic score has been classified into the following 4 categories: 0 , normal or inactive disease; 1 , mild disease with erythema, decreased vascular patterns and mild friability; 2, moderate disease with marked erythema, absence of vascular patterns, friability and erosions; and 3, severe disease with spontaneous bleeding and ulceration [12].

Outcomes assessed in the analysis were as follows:

1. Pooled rate of clinical remission, defined as: CAI score $\leq 4$; Ulcerative Colitis Disease Activity Index (UCDAI) $\leq 2$ or $<3$, SCCAI $\leq 2$.

2. Pooled rate of clinical response, defined as: decrease in UCDAI by $\geq 3$; decrease in partial Mayo score by $\geq 3$; and decrease in SCCAI score by $\geq 3$ points.

3. Pooled rate of endoscopic response and remission, defined as: drop in Mayo score $\geq 1$ to a score of 0 or 1 for remission and any $\geq 1$ in Mayo sub-score as response as well as a partial Mayo score $\leq 1$.

4. Safety profile, including adverse events.

\section{Statistical analysis}

We used meta-analysis techniques to calculate the pooled estimates in each case, using a random-effects model and 
following the methods suggested by DerSimonian and Laird [13]. When the incidence of an outcome was 0 in a study, a continuity correction of 0.5 was added to the number of incident cases before statistical analysis [14]. We assessed heterogeneity between study-specific estimates using the Cochran Q statistical test for heterogeneity, 95\% prediction interval (PI), which deals with the dispersion of the effects [15-17], and the $I^{2}$ statistics $[18,19]$. In this, values of $<30 \%, 30-60 \%, 61-75 \%$, and $>75 \%$ were suggestive of low, moderate, substantial, and considerable heterogeneity, respectively [20]. Publication bias was ascertained, qualitatively by visual inspection of a funnel plot and quantitatively by the Egger test [21]. When publication bias was present, further statistics using the fail-safe $\mathrm{N}$ test and Duval and Tweedie's "Trim and Fill" test was used to ascertain the impact of the bias [22]. Three levels of impact were reported, based on the concordance between the reported results and the actual estimate if there were no bias. The impact was reported as minimal if both versions were estimated to be same, modest if effect size changed substantially but the final finding would still remain the same, and severe if the basic final conclusion of the analysis was threatened by the bias [23]. Predictive factors for the outcomes were assessed by meta-regression methods. All analyses were performed using Comprehensive MetaAnalysis (CMA) software, version 3 (BioStat, Englewood, NJ).

\section{Results}

\section{Search results and population characteristics}

From an initial total of 119 studies, 101 records were screened and 74 full-length articles were assessed. Seven studies (380 patients) were included in the final analysis [24-30]: 188 patients were treated with curcumin as an adjunct to mesalamine and 192 patients were in the control group, receiving placebo with mesalamine. One study [25] reported clinical outcomes using the CAI, 2 studies $[29,26]$ used the UCDAI, 2 studies [30,24] used the Mayo/partial Mayo score, and $2[27,28]$ used the SCCAI. The schematic diagram of study selection is illustrated in Supplementary Fig. 1.

There were 174 males and 128 females. Two studies did not report the patients' sex. Mean age ranged from 32.7 \pm 8.9 years to $45.2 \pm 15.8$ years. The basic population characteristics are described in Supplementary Table 2. Four studies [29,26,28,27] reported the extent of colitis: left sided colitis (52 patients), pancolitis (24 patients), and proctitis (28 patients). In 6 studies [24-28,30], oral curcumin was used, whereas in 1 study [29] the route of administration was rectal. In the study by Masoodi et al [28], the actual number of patients who achieved an overall final clinical response was not reported. As the study was otherwise of high quality, the authors decided to include it in the analysis. The primary author of the study was contacted, but it was not possible to obtain the missing information and the most appropriate data were extracted. The potential influence of this study on outcomes, if any, was evaluated using a sensitivity analysis.

\section{Characteristics and quality of included studies}

All included studies were RCTs. Two studies were published in abstract form $[24,30]$ and the rest as full manuscripts. The detailed assessment of study quality is given in Supplementary Table 1. Overall, all studies were considered to be of high quality based on the Jadad scale. There were no low-quality studies.

\section{Meta-analysis outcomes}

The pooled odds ratio for clinical remission (5 studies) [25-27,29,30] with curcumin use was 2.9 (95\% confidence interval [CI] 1.5-5.5, 95\% prediction interval [PI] 0.5-33, $I^{2}=45, \mathrm{P}=0.002$ ) (forest plot Fig. 1), while for clinical response with curcumin (5 studies) [24,26-29] it was 2.6 (95\%CI 1.5-4.5, 95\%PI 0-88, $I^{2}=74 \%, \mathrm{P}=0.001$ ) (forest plot Fig. 2). The pooled odds ratio for endoscopic response/ remission (5 studies) $[24,26,27,29,30]$ was 2.3 (95\%CI 1.2-4.6, 95\%PI 0-14, $I^{2}=35.5 \%, \mathrm{P}=0.01$ ) (forest plot Fig. 3).

With regards to safety and adverse events, Lang et al [27] reported 3 serious adverse events resulting in withdrawal of the subjects from the study. Two patients reported worsening UC symptoms and 1 patient reported abdominal pain from a peptic ulcer present prior to initiation of the study medication. Four patients reported mild adverse events, such as nausea, transient increase in stool frequency and abdominal bloating.

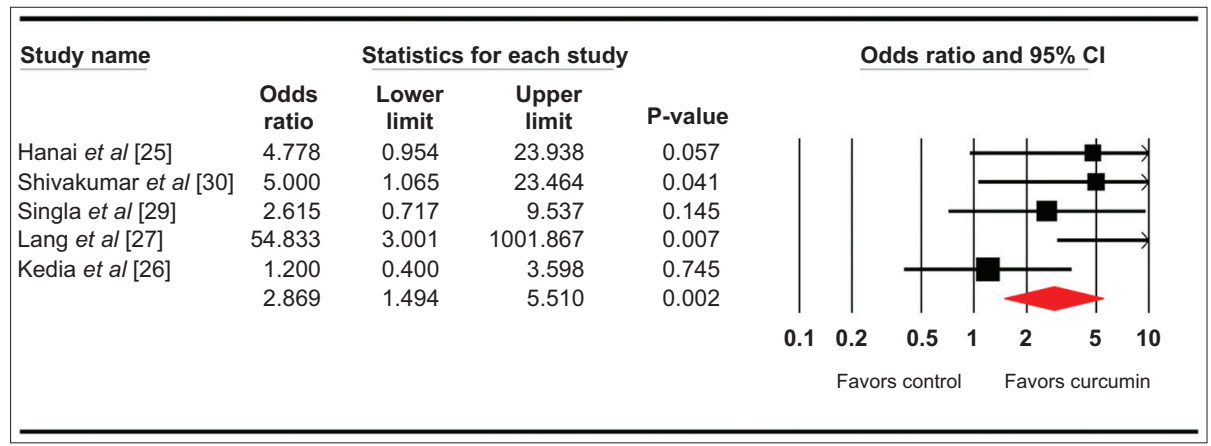

Figure 1 Forest plot. Clinical remission CI, confidence interval 
Nine adverse events were reported in 7 patients by Hanai et al [25], including sensation of abdominal distension, nausea, transient hypertension, transient increase in the number of stools and elevated serum guanosine triphosphate level. Masoodi et al [28] reported a total of 8 adverse events, including flatulence, dyspepsia, headache, increased appetite, nausea and yellow stools. There were no serious adverse events.

Meta-regression analysis was done based on the curcumin dosage used. The lowest dose used was $100 \mathrm{mg}$ and the maximum $10000 \mathrm{mg}$. No significant predictive effect was noted with curcumin dosage on the calculated outcomes (Random effects KnappHartung 2-sided P-value=0.54, 0.34, 0.66 for clinical remission, clinical response and endoscopic response, respectively).

\section{Validation of meta-analysis results}

\section{Sensitivity analysis}

To assess whether any one study had a dominant effect on the meta-analysis, we excluded one study at a time and analyzed its effect on the main summary estimate. On this analysis, no single study significantly affected the outcome or the heterogeneity. Thus, removing the study by Masoodi et al [28], would not have changed our findings.

\section{Heterogeneity}

We assessed the dispersion of the calculated rates using the PI and $I^{2}$ percentage values. The PI gives an idea of the range of the dispersion and $I^{2}$ tells us what proportion of the dispersion is true versus chance [17]. The pooled rates of primary outcomes had wide prediction intervals with heterogeneity.

\section{Publication bias}

A publication bias analysis was not done, as the total number of studies included in the analysis was less than 10 .

\section{Discussion}

Our study demonstrates that adjunctive use of curcumin with mesalamine yields a superior clinical and endoscopic response in the treatment of UC compared to placebo and mesalamine. This study is the first meta-analysis to report on the use of curcumin as an adjunct to mesalamine in the treatment of UC, and it is the most comprehensive review to date of all human trials evaluating the use of adjunctive curcumin therapy in treating UC.

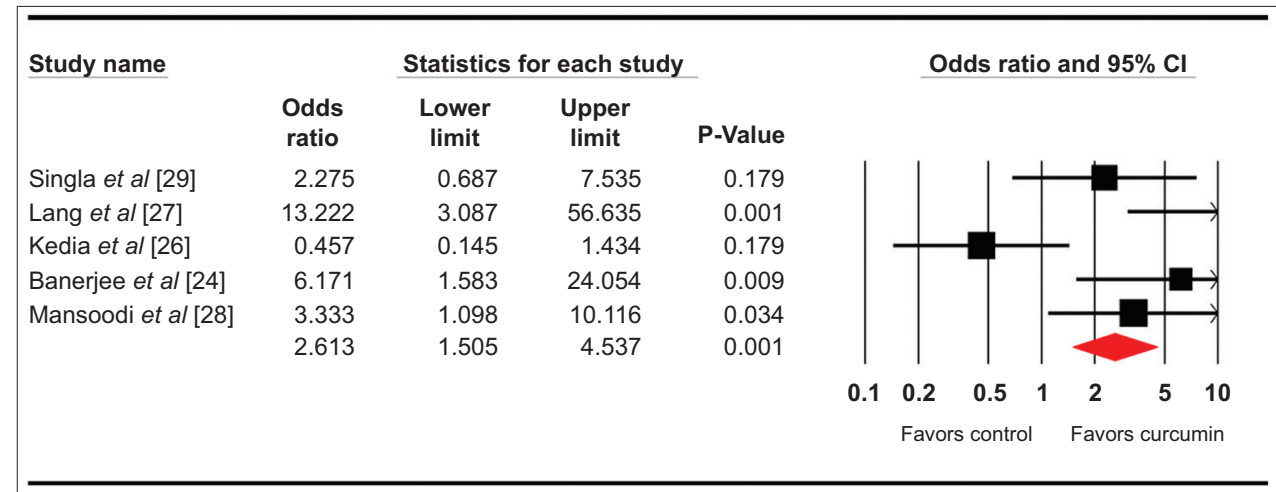

Figure 2 Forest plot. Clinical response CI, confidence interval

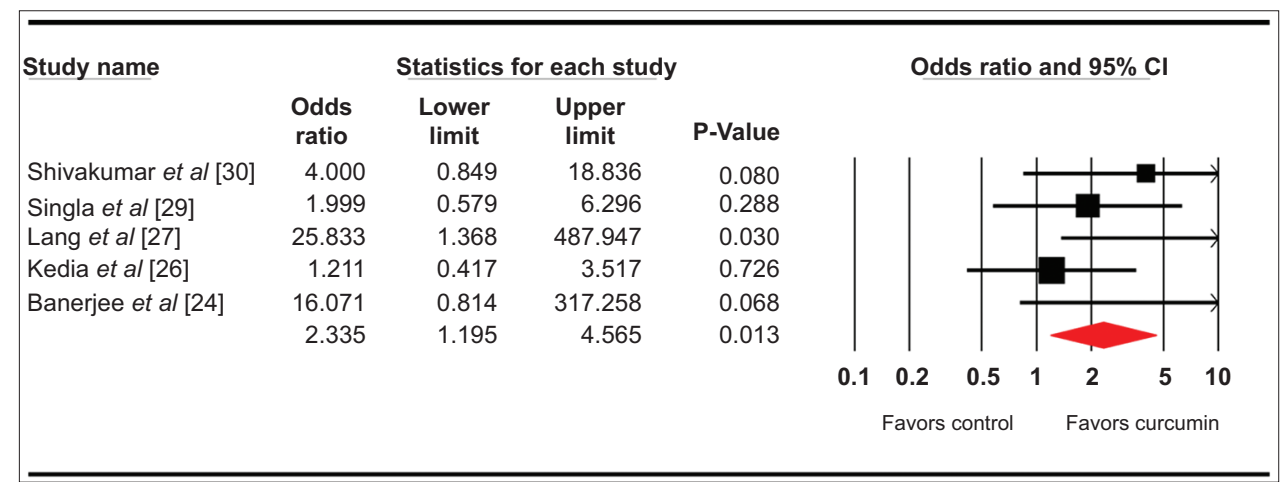

Figure 3 Forest plot. Endoscopic response CI, confidence interval 
Based on our meta-analysis of clinical trials, the odds of a clinical and endoscopic response to curcumin as an adjunct to mesalamine, compared to placebo with mesalamine, were approximately threefold. A total of 21 adverse events were reported, as mentioned in the Results section, all of which were of mild degree.

In experimental models, curcumin has been shown to prevent colitis induced by tri-nitro-benzene sulfonic acid and dextran sodium sulfate. Its postulated mechanism of action is suppression of nuclear factor k-light chain enhancer in B-lymphocytes, along with favorable expression of Th1 and Th2 cytokines. Curcumin has also been reported to have antiinterleukin-1 and anti-tumor necrosis factor a properties, which makes it an attractive naturopathic treatment option for inflammatory diseases such as UC [31,32].

The results of this study are on par with the reported outcomes on the use of curcumin in UC published in the literature $[28,25,27,33]$. One negative study, that by Kedia et al [26], reported no significant differences in the rates of clinical remission, clinical response, mucosal healing, and treatment failure between curcumin and placebo at 8 weeks of treatment. Discrepancies in the drug dosage, drug delivery and duration of treatment are some of the postulated reasons for this outlier. Curcuminoids are lipophilic molecules and their absorption in the gastrointestinal tract can be low and variable [34]. Currently, we do not know whether the therapeutic effects of curcumin depend on its absorption and systemic bioavailability, or are more the result of a topical action on the intestinal mucosa. Clearly, there exists a significant "knowledge gap" regarding the use of curcumin in human beings.

The strengths of this review are as follows: systematic literature search with well-defined inclusion criteria, careful exclusion of redundant studies, inclusion of good quality studies with detailed extraction of data, rigorous evaluation of study quality, and statistics to establish and/or refute the validity of the results of our meta-analysis. We report the prediction intervals, thereby making our comparative pooled estimates applicable to the real population.

There were limitations to this study, most of which are inherent to any meta-analysis. The included studies were not entirely representative of the general population and community practice, with most being performed in tertiary-care referral centers. Heterogeneity was noted, most probably due to differences in the severity scoring indices used, the dosages of curcumin and the route of drug delivery. We wanted to analyze all the published literature on curcumin use in UC and included studies with active, quiescent as well as mild-to-moderate disease activity. We were not able to analyze our results based on the presence of comorbidities and were not able to assess the predictors of treatment success and/or failure. Nevertheless, our study is the best available estimate in the literature thus far with respect to the use of curcumin as an adjunct to mesalamine in the treatment of UC.

In conclusion, our meta-analysis demonstrates that curcumin, when combined with mesalamine, yields a superior clinical and/or endoscopic response in UC, albeit with heterogeneity. Given the minimal adverse events, we recommend that curcumin be considered as an adjunct to mesalamine in the treatment of UC.

\section{Acknowledgment}

The authors would like to thank Emily J. Glenn, MSLS (Education and research services, McGoogan Library of Medicine, University of Nebraska Medical Center) for help with the literature and database search

\section{Summary Box}

\section{What is already known:}

- American Gastroenterology Association guidelines recommend starting standard dose mesalamine or diazo-bonded 5-amino-salicylic acid (5-ASA) in patients with extensive mild-tomoderate ulcerative colitis (UC)

- Rectal mesalamine is recommended in addition to oral 5-ASA in patients with extensive or left-sided mild-to-moderate UC

- No recommendation was made on the use of curcumin in mild-to-moderate UC patients

\section{What the new findings are:}

- Based on this meta-analysis of randomized controlled trials, combination therapy of curcumin with mesalamine in patients with mildto-moderate UC yields a superior clinical and endoscopic response

- Odds ratio (OR) for clinical remission with curcumin was 2.9 (95\% confidence interval [CI] 1.5-5.5), $\mathrm{P}=0.002$; OR for a clinical response with curcumin was 2.6 (95\%CI 1.5-4.5), $\mathrm{P}=0.001$; OR for an endoscopic response and/or remission with curcumin was 2.3 (95\%CI 1.2-4.6), $\mathrm{P}=0.01$

\section{References}

1. Langholz E, Munkholm P, Nielsen OH, Kreiner S, Binder V. Incidence and prevalence of ulcerative colitis in Copenhagen county from 1962 to 1987. Scand J Gastroenterol 1991;26:12471256.

2. Langholz E, Munkholm P, Davidsen $\mathrm{M}$, Nielsen $\mathrm{OH}$, Binder V. Changes in extent of ulcerative colitis: a study on the course and prognostic factors. Scand J Gastroenterol 1996;31:260-266.

3. Singh S, Feuerstein JD, Binion DG, Tremaine WJ.AGA technical review on the management of mild-to-moderate ulcerative colitis. Gastroenterology 2019;156:769-808.

4. Panahi Y, Khalili N, Sahebi E, et al. Effects of curcuminoids plus piperine on glycemic, hepatic and inflammatory biomarkers in 
patients with type 2 diabetes mellitus: a randomized double-blind placebo-controlled trial. Drug Res (Stuttg) 2018;68:403-409.

5. Panahi Y, Kianpour P, Mohtashami R, Jafari R, Simental-Mendía LE, Sahebkar A. Curcumin lowers serum lipids and uric acid in subjects with nonalcoholic fatty liver disease: a randomized controlled trial. J Cardiovasc Pharmacol 2016;68:223-229.

6. Sahebkar A, Serban MC, Gluba-Brzózka A, et al. Lipid-modifying effects of nutraceuticals: An evidence-based approach. Nutrition 2016;32:1179-1192.

7. Moher D, Liberati A, Tetzlaff J, Altman DG; PRISMA Group. Preferred reporting items for systematic reviews and metaanalyses: the PRISMA statement. Ann Intern Med 2009;151:264269, W64.

8. Jadad AR, Moore RA, Carroll D, et al. Assessing the quality of reports of randomized clinical trials: is blinding necessary? Control Clin Trials 1996;17:1-12.

9. Rachmilewitz D. Coated mesalazine (5-aminosalicylic acid) versus sulphasalazine in the treatment of active ulcerative colitis: a randomised trial. BMJ 1989;298:82-86.

10. Walmsley RS, Ayres RC, Pounder RE, Allan RN. A simple clinical colitis activity index. Gut 1998;43:29-32.

11. D'Haens G, Sandborn WJ, Feagan BG, et al. A review of activity indices and efficacy end points for clinical trials of medical therapy in adults with ulcerative colitis. Gastroenterology 2007;132:763-786.

12. Schroeder KW, TremaineWJ, Ilstrup DM.Coated oral5-aminosalicylic acid therapy for mildly to moderately active ulcerative colitis. A randomized study. N Engl J Med 1987;317:1625-1629.

13. DerSimonian R, Laird N. Meta-analysis in clinical trials. Control Clin Trials 1986;7:177-188.

14. Sutton AJ, Abrams KR, Jones DR, et al. Methods for meta-analysis in medical research. John Wiley \& Sons Ltd.: New York; 2000, pp. 205-228.

15. Higgins J, Thompson SG, Spiegelhalter DJ. A re-evaluation of random-effects meta-analysis. $J$ R Stat Soc Ser A Stat Soc 2009; 172:137-159.

16. Riley RD, Higgins JP, Deeks JJ. Interpretation of random effects meta-analyses. BMJ 2011;342:d549.

17. Mohan BP, Adler DG. Heterogeneity in systematic review and meta-analysis: how to read between the numbers. Gastrointest Endosc 2019;89:902-903.

18. Kanwal F, White D. "Systematic Reviews and Meta-analyses" in Clinical Gastroenterology and Hepatology. Clin Gastroenterol Hepatol 2012;10:1184-1186.

19. Higgins JP, Thompson SG, Deeks JJ, Altman DG. Measuring inconsistency in meta-analyses. BMJ 2003;327:557-560.

20. Guyatt GH, Oxman AD, Kunz R, et al. GRADE guidelines: 7 . Rating the quality of evidence-inconsistency. J Clin Epidemiol
2011;64:1294-1302.

21. Easterbrook PJ, Berlin JA, Gopalan R, Matthews DR. Publication bias in clinical research. Lancet 1991;337:867-872.

22. Duval S, Tweedie R. Trim and fill: A simple funnel-plot-based method of testing and adjusting for publication bias in metaanalysis. Biometrics 2000;56:455-463.

23. Rothstein HR, Sutton AJ, Borenstein M. Publication bias in metaanalysis: Prevention, assessment and adjustments. John Wiley \& Sons; 2006.

24. Banerjee R, Medaboina K, Boramma GG, et al. Novel bio-enhanced curcumin with mesalamine for induction of remission in mild to moderate ulcerative colitis. Gastroenterology 2017;152:S587.

25. Hanai $H$, Iida $T$, Takeuchi $K$, et al. Curcumin maintenance therapy for ulcerative colitis: randomized, multicenter, doubleblind, placebo-controlled trial. Clin Gastroenterol Hepatol 2006;4:1502-1506.

26. Kedia S, Bhatia V, Thareja S, et al. Low dose oral curcumin is not effective in induction of remission in mild to moderate ulcerative colitis: Results from a randomized double blind placebo controlled trial. World J Gastrointest Pharmacol Ther 2017;8:147-154.

27. Lang A, Salomon N, Wu JC, et al. Curcumin in combination with mesalamine induces remission in patients with mild-tomoderate ulcerative colitis in a randomized controlled trial. Clin Gastroenterol Hepatol 2015;13:1444-1449.e1.

28. Masoodi M, Mahdiabadi MA, Mokhtare M, et al. The efficacy of curcuminoids in improvement of ulcerative colitis symptoms and patients' self-reported well-being: A randomized double-blind controlled trial. J Cell Biochem 2018;119:9552-9559.

29. Singla V, Pratap Mouli V, Garg SK, et al. Induction with NCB-02 (curcumin) enema for mild-to-moderate distal ulcerative colitis - a randomized, placebo-controlled, pilot study. J Crohns Colitis 2014;8:208-214.

30. Shivakumar V, Sharonjeet K, Dutta U, et al. A double blind randomised controlled trial to study the effect of oral Curcumina longa versus placebo in patients with active ulcerative colitis. Indian J Gastroenterol 2011;30(Suppl 1):A37. [Abstract]

31. Arbuckle TE, Fraser WD, Fisher M, et al. Cohort profile: the maternal-infant research on environmental chemicals research platform. Paediatr Perinat Epidemiol 2013;27:415-425.

32. Sahebkar A, Cicero AFG, Simental-Mendía LE, Aggarwal BB, Gupta SC. Curcumin downregulates human tumor necrosis factor- $\alpha$ levels: a systematic review and meta-analysis of randomized controlled trials. Pharmacol Res 2016;107:234-242.

33. Holt PR, Katz S, Kirshoff R. Curcumin therapy in inflammatory bowel disease: a pilot study. Dig Dis Sci 2005;50:2191-2193.

34. Shakeri A, Panahi Y, Johnston TP, et al. Biological properties of metal complexes of curcumin. Biofactors 2019;45:304-317. 


\section{Supplementary material}

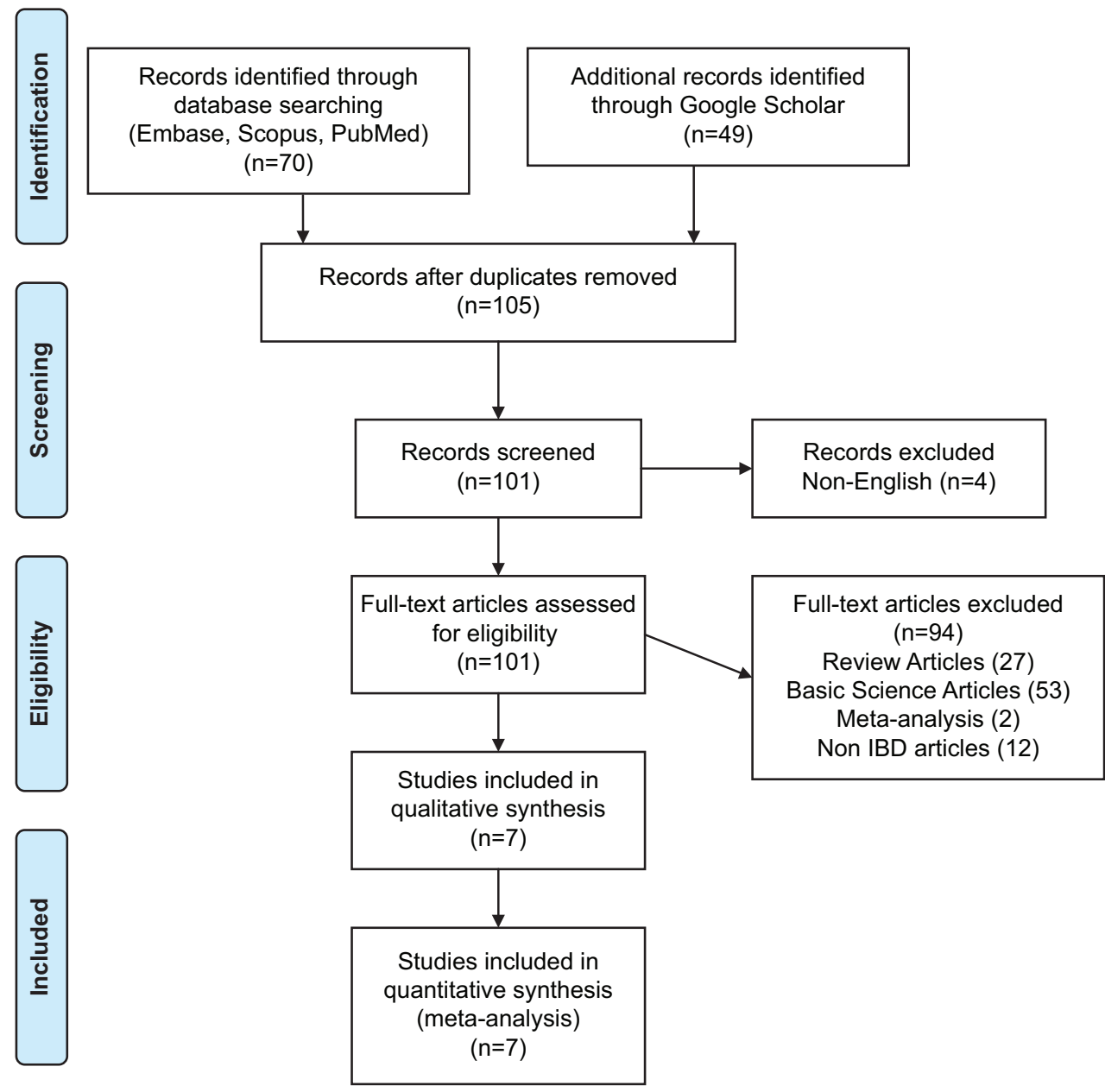

Supplementary Figure 1 PRISMA study selection PRISMA 2009 Flow Diagram

Source: Moher D, Liberati A, Tetzlaff J, Altman DG, The PRISMA Group. Preferred Reporting Items for Systematic Reviews and Meta-Analyses: The PRISMA Statement. PLoS Med 2009;6:6(7): e1000097. doi:10.1371/journal.pmed1000097

Supplementary Table 1 Jadad study quality assessment

\begin{tabular}{|c|c|c|c|c|c|c|c|}
\hline Study & Hanai & Shivakumar & Singla & Lang & Kedia & Banerjee & Masoodi \\
\hline \multicolumn{8}{|l|}{ Randomization } \\
\hline Randomization mentioned: +1 & 1 & 1 & 1 & 1 & 1 & 1 & 1 \\
\hline Randomization appropriate: +1 & 1 & 1 & 1 & 1 & 1 & 1 & 1 \\
\hline $\begin{array}{l}\text { Inappropriate method of } \\
\text { randomization: }-1\end{array}$ & - & - & - & - & - & - & - \\
\hline \multicolumn{8}{|l|}{ Blinding } \\
\hline Blinding mentioned: +1 & 1 & 1 & 1 & 1 & 1 & 1 & 1 \\
\hline Method appropriate: +1 & 1 & 1 & 1 & 1 & 1 & 1 & 1 \\
\hline Method inappropriate: -1 & - & - & - & - & - & - & - \\
\hline \multicolumn{8}{|l|}{ Account of all patients } \\
\hline All pts accounted for: +1 & 1 & 1 & 1 & 1 & 1 & 1 & 1 \\
\hline Score & 5 & 5 & 5 & 5 & 5 & 5 & 5 \\
\hline Quality & High & High & High & High & High & High & High \\
\hline
\end{tabular}




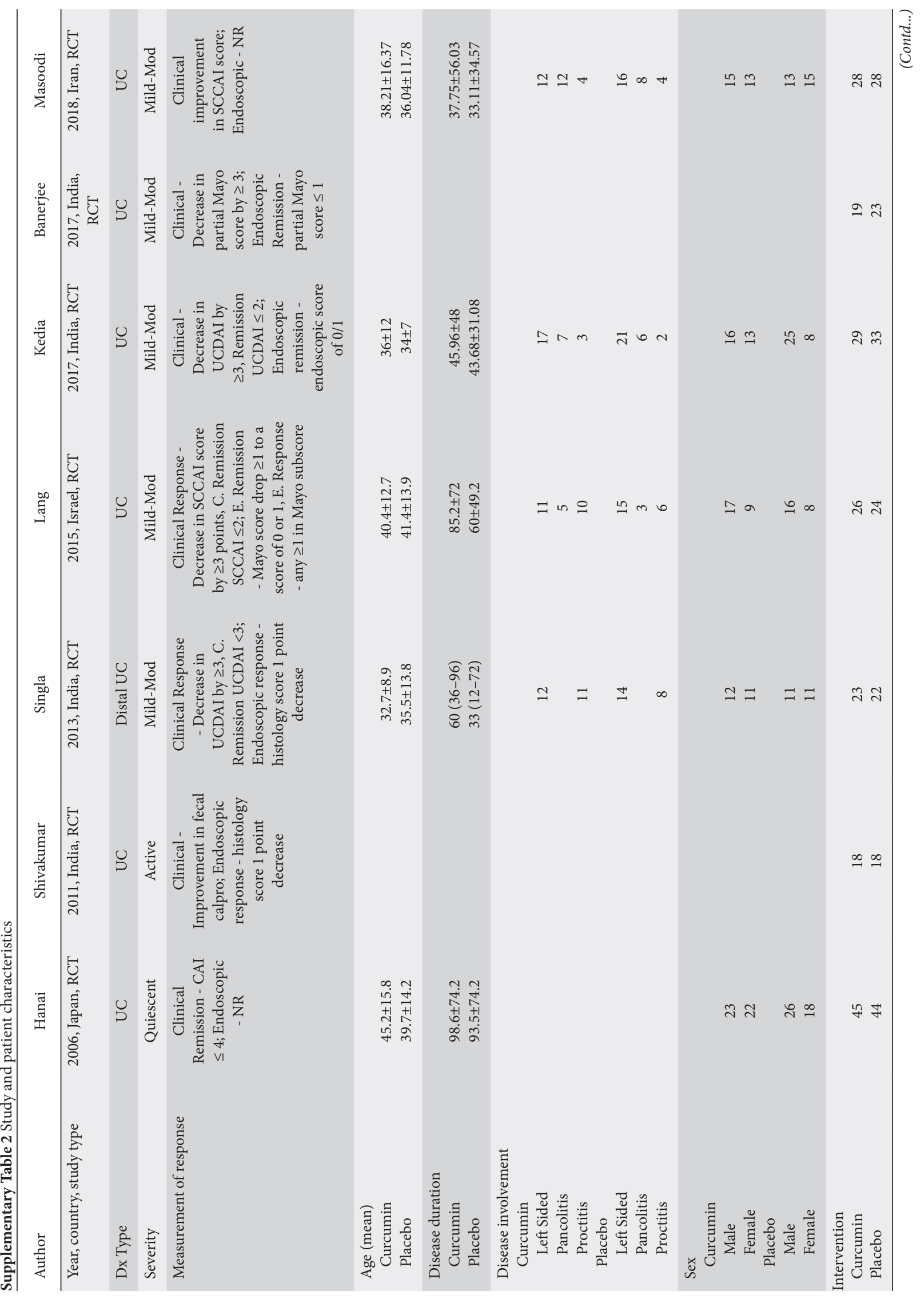




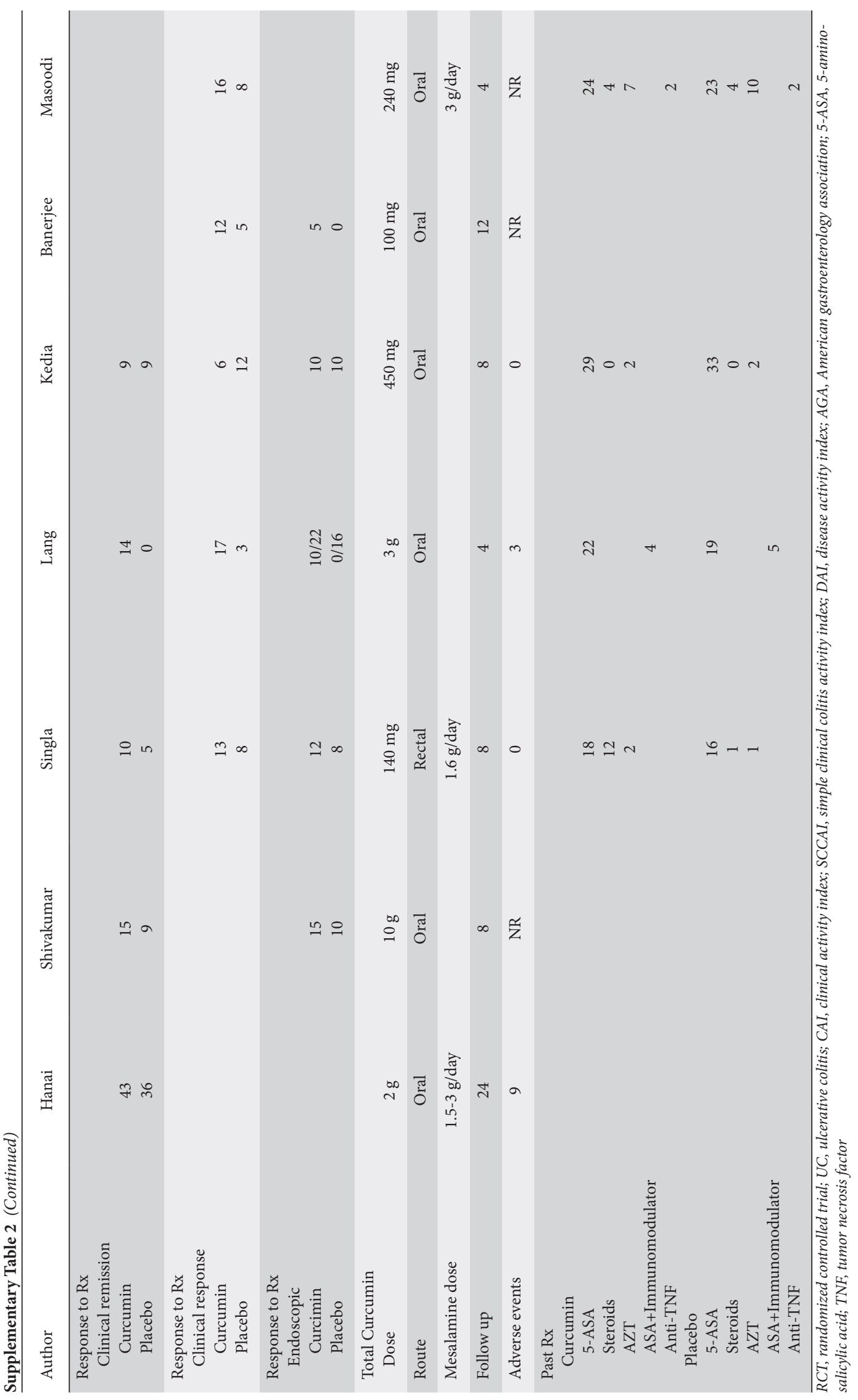

